Case Report

\title{
Case Report: Homozygous C677T MTHFR Gene Mutation in Male with Hypogonadism
}

\author{
Rami Salameh (D), Mumtaheena Miah, and Catherine Anastasopoulou \\ Endocrinology Department, Albert Einstein Medical Center-Philadelphia, Philadelphia, PA, USA \\ Correspondence should be addressed to Rami Salameh; rami_salameh@hotmail.com
}

Received 21 May 2019; Accepted 17 March 2020; Published 19 May 2020

Academic Editor: Hyunsik Kang

Copyright ( 92020 Rami Salameh et al. This is an open access article distributed under the Creative Commons Attribution License, which permits unrestricted use, distribution, and reproduction in any medium, provided the original work is properly cited.

\begin{abstract}
We report a 44-year-old male, who was diagnosed with hypogonadotropic hypogonadism after complaining of erectile dysfunction, depression, and fatigue. He was started on testosterone replacement therapy. He persistently complained of fatigue despite increasing the dose of testosterone over two years and having therapeutic testosterone levels. He was found to have homozygous C677T methylenetetrahydrofolate reductase (MTHFR) gene mutation. After treatment with folate and B12, his symptoms resolve completely. MTHFR is a key enzyme in the folate pathway, and it plays an essential role in homocysteine metabolism. Homozygous C677T individuals have decreased activity of MTHFR enzyme with increased homocysteine levels, which is associated with increased risk of thrombosis. An association has been reported between C677T variant and male infertility. Patients identified to have hyperhomocysteinemia should be treated with B-complex vitamin supplements. Our case emphasizes other possible etiologies for fatigue and erectile dysfunction in a male with hypogonadism on testosterone therapy. Also, it shows possible association between MTHFR gene mutation and male hypogonadism.
\end{abstract}

\section{Introduction}

MTHFR is a key enzyme in the folate pathway, and it plays an essential role in homocysteine metabolism. Homozygous C677T individuals have decreased activity of MTHFR enzyme with potentially increased homocysteine levels that are associated with increased risk of venous thrombosis, stroke, coronary artery disease, and recurrent pregnancy loss in females. There have also been reports of association between C677T variant and male infertility.

\section{Case Report}

We report a case of a 44-year-old male, who was a semiprofessional football player in late teenage years, with a history of multiple head concussion between age 15 and 25 .

He was diagnosed with hypogonadotropic hypogonadism in 2013, when he complained of erectile dysfunction, depression, generalized fatigue. He was started on testosterone replacement therapy. He persistently complained of fatigue despite increasing the dose of testosterone over two years and having therapeutic testosterone levels on labs while on therapy.

At that point, he underwent genetic testing, as per patient personal request, which showed homozygous C677T MTHFR gene mutation.

Other relevant labs included folate: $3.1 \mathrm{ng} / \mathrm{mL}$ (normal range: 4.2-19.9) and $\mathrm{B} 12: 333 \mathrm{pg} / \mathrm{mL}$ (normal range: 210-934).

His hemoglobin was at normal levels, but interestingly, it did not increase as expected with the use of testosterone.

The patient was treated with folate and B12 supplements, which made his symptoms resolve completely (Table 1).

\section{Discussion}

MTHFR is a key enzyme in the folate pathway, and it plays an essential role in homocysteine metabolism, as shown in Figure 1.

Homozygous C677T individuals have decreased activity of MTHFR enzyme, thus having an increased risk for hyperhomocysteinemia when deficient in folate, vitamin 
TABLe 1: Social history.

Smoking

Alcohol use

Recreational drug use

Physical activity

BMI
Current smoker, 10 pack-year

Occasional; a glass of beer or wine on occasions None/never

Exercises for 1 hour 3 days a week; walking or weightlifting

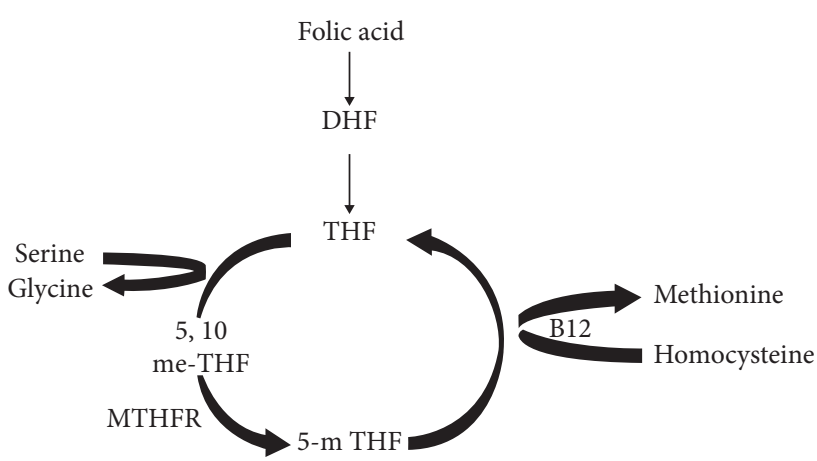

Figure 1: Folic acid is reduced to dihydrofolate (DHF) then to tetrahydrofolate (THF). THF is converted to 5,10-methylenetetrahydrofolate $(5,10-\mathrm{me}-\mathrm{THF})$, which is then reduced to 5methyltetrahydrofolate (5-mTHF) by MTHFR. 5-mTHF is demethylated to complete the folate cycle by donating a carbon into the methionine cycle through the methylation of homocysteine to methionine by methionine synthase and its cofactor vitamin B12.

B12, or pyridoxine [1]. Elevated homocysteine levels are associated with increased risk of venous thromboembolism, strokes, coronary artery disease, and recurrent pregnancy loss [2]. The exact cause of accelerated vascular disease is unclear, but several mechanisms have been suggested, including endothelial cell damage, lipid peroxidation, smooth muscle cell proliferation, downregulation of antithrombotic factors, and upregulation of prothrombotic factors.

Male hypogonadism, defined as decreased testosterone production and/or impaired ability to produce functional sperms, overlaps with male infertility. The association between C677T variant and male infertility has been reported in the literature.

While some studies did not find an association between C677T polymorphism and male infertility $[3,4]$, Bezold et al. [5] reported that the prevalence of the homozygous C677T mutation was $18.8 \%$ in infertile males as compared with 9.5\% in normal fertile males. Thus, it was concluded that MTHFR products may play a role in the pathogenesis of male infertility

Mfady et al. [6] showed that in Jordanians, MTHFR C677T polymorphism was associated with male infertility, while MTHFR A1298C was not. Similar results were reported by Park et al. [7] in the South Korean population.

Gong et al. [8] conducted a meta-analysis on 26 studies involving 5,575 cases and 5,447 controls. MTHFR C677T polymorphism was significantly associated with male infertility in Asians as well as in Caucasians.

Other studies, on the other hand, concluded that there is an association between C677T variant and male infertility in Indian, African, and Southeast Asian populations. Data from
Europe regarding this association are conflicting. The lack of similar association in Western populations could be due to the overall dietary enrichment with folates [9].

Lombardo et al. [10] studied the role of C677T MTHFR mutation with subsequent hyperhomocysteinemia in erectile dysfunction. He concluded that hyperhomocysteinemia in homozygous C677T patients may interfere with erection mechanisms and thus cause erectile dysfunction.

Patients identified to have hyperhomocysteinemia should be treated with B-complex vitamin supplements. Initiation of therapy with folic acid, B12, and B6 vitamins tends to normalize homocysteine in 1-2 months. It is unclear whether normalization of homocysteine would improve male infertility or not.

\section{Conclusion}

Our case emphasizes other important etiologies for fatigue and erectile dysfunction in a male with hypogonadism on testosterone therapy as well as shows the possible association between MTHFR gene mutation and male hypogonadism.

\section{Disclosure}

This abstract was presented as a poster in AACE 2019 Meeting in Los Angeles.

\section{Conflicts of Interest}

The authors declare that they have no conflicts of interest.

\section{References}

[1] P. F. Jacques, A. G. Bostom, R. R. Williams et al., "Relation between folate status, a common mutation in methylenetetrahydrofolate reductase, and plasma homocysteine concentrations," Circulation, vol. 93, no. 1, pp. 7-9, 1996.

[2] S.-C. Liew and E. D. Gupta, "Methylenetetrahydrofolate reductase (MTHFR) C677T polymorphism: epidemiology, metabolism and the associated diseases," European Journal of Medical Genetics, vol. 58, no. 1, pp. 1-10, 2015.

[3] I. M. W. Ebisch, W. L. van Heerde, C. M. G. Thomas, N. van der Put, W. Y. Wong, and R. P. M. Steegers-Theunissen, "C677T methylenetetrahydrofolate reductase polymorphism interferes with the effects of folic acid and zinc sulfate on sperm concentration," Fertility and Sterility, vol. 80, no. 5, pp. 1190-1194, 2003.

[4] L. Stuppia, V. Gatta, O. Scarciolla et al., "The methylenetethrahydrofolate reductase (MTHFR) C677T polymorphism and male infertility in Italy," Journal of Endocrinological Investigation, vol. 26, no. 7, pp. 620-622, 2003.

[5] G. Bezold, M. Lange, and R. U. Peter, "Homozygous methylenetetrahydrofolate reductase C677T mutation and male 
infertility," New England Journal of Medicine, vol. 344, no. 15, pp. 1172-1173, 2001.

[6] D. S. Mfady, M. F. Sadiq, O. F. Khabour, A. S. Fararjeh, A. Abu-Awad, and Y. Khader, "Associations of variants in MTHFR and MTRR genes with male infertility in the Jordanian population," Gene, vol. 536, no. 1, pp. 40-44, 2014.

[7] J. H. Park, H. C. Lee, Yu-Mi Jeong et al., "MTHFR C677T polymorphism associates with unexplained infertile male factors," Journal of Assisted Reproduction and Genetics, vol. 22, no. 9-10, pp. 361-368, 2005.

[8] M. Gong, W. Dong, T. He et al., "MTHFR 677C> T polymorphism increases the male infertility risk: a meta-analysis involving 26 studies," PLoS One, vol. 10, no. 3, 2015.

[9] K. Singh, S. K. Singh, R. Sah, I. Singh, and R. Raman, "Mutation C677T in the methylenetetrahydrofolate reductase gene is associated with male infertility in an Indian population1," International Journal of Andrology, vol. 28, no. 2, pp. 115-119, 2005.

[10] F. Lombardo, P. Tsamatropoulos, E. Piroli et al., "Treatment of erectile dysfunction due to C677T mutation of the MTHFR gene with vitamin $\mathrm{B} 6$ and folic acid in patients non responders to PDE5i," The Journal of Sexual Medicine, vol. 7, no. 1, pp. 216-223, 2010. 